\title{
ROTATIONAL COHERENCE SPECTROSCOPY AND EXCITED-STATE DYNAMICS OF TOLANE AND ITS VAN DER WAALS COMPLEXES WITH ARGON AND NITROGEN
}

\author{
SHANE M. OHLINE, JOANN ROMASCAN, and PETER M. FELKER \\ Department of Chemistry and Biochemistry, University of California, Los \\ Angeles, CA 90024-1569
}

(Received 4 April 1993)

\begin{abstract}
We report the results of rotational coherence spectroscopy on tolane, tolane-Ar, and tolane- $\mathrm{N}_{2}$. The results on tolane are consistent with a planar geometry for the species. They also provide information about the symmetries of excited vibronic states in the species. The results on the van der Waals complexes provide significant experimental constraints on where the small species bind to tolane. In addition, we report the observation of a rapid excited-state decay process in the complexes and discuss the possible nature of this process.
\end{abstract}

KEY WORDS: Rotational coherence, tolane complexes, excited-state dynamics

\section{INTRODUCTION}

The study of large species by supersonic molecular beam spectroscopies has been a theme of Professor Mitsuo Ito's for the past decade. ${ }^{1}$ One of the species studied by his group with such techniques is the tolane molecule (diphenyl acetylene). ${ }^{2}$ Their interest in this species was based primarily on characterizing its large amplitude torsional motions and the potential energy surfaces that govern such motions. We have become interested in tolane because it can serve usefully as a complexing partner in weakly bound van der Waals complexes. As is well known, the study of such species is a fruitful source of information on the nature of intermolecular interactions.

In this paper we report the results of spectroscopic experiments on jet-cooled tolane, tolane-Ar, and tolane- $\mathrm{N}_{2}$. The principal aim of the study has been to obtain structural information about these species. Toward this end we have used rotational coherence spectroscopy (RCS). ${ }^{3}$ RCS is a picosecond time-domain method of rotational spectroscopy that is particularly advantageous in structural studies of large species. With RCS we have measured rotational constants of bare tolane and have 
found that they are consistent with a planar structure for the species. The RCS results on bare tolane also provide information pertaining to the transition-moment directions of several vibronic bands of the species. This information suggests an assignment for the electronic structure of the molecule that is consistent with that of Reference 2. From the RCS results on tolane-Ar and tolane- $\mathrm{N}_{2}$ information about where the smaller species bind to tolane has been obtained. Besides this, we have observed fast decays for the excited states of both these complexes, decays that either reflect vibronic energy redistribution processes or predissociation.

The paper is composed of the following sections. In section II we outline the experimental procedures. Section III presents the experimental results. In section IV an analysis of the structures of the species, as derived from the RCS results, is given. Finally, section $\mathrm{V}$ is a discussion of two topics: the assignment of vibronic bands in bare tolane, and the structure and dynamics of tolane-Ar and tolane- $\mathrm{N}_{2}$.

\section{EXPERIMENTAL SECTION}

RCS was implemented by means of the time-resolved fluorescence depletion (TRFD) scheme $\mathrm{e}^{4}$ of picosecond spectroscopy. The apparatus and general procedures have been described in detail elsewhere. ${ }^{5}$ Here, we describe only those specifics pertinent to the tolane experiments. The excitation pulses for TRFD were produced by a picosecond laser system composed of a Q-switched, mode-locked CW Nd:YAG laser and a cavity-dumped dye laser. The fundamental of the dye laser (Rhodamine 590 in methanol as dye) was frequency doubled in $\beta$-barium borate to produce excitation pulses in the range between $35,051 \mathrm{~cm}^{-1}$-the " $a$ " band of tolane-and $35,589 \mathrm{~cm}^{-1}$-the " $B$ "' band (the frequencies and notation are from Reference 2). The frequency-doubled dye-laser output was directed through a Michelson interferometer to produce the pump and variably delayed probe pulse trains. The pump and probe pulses were linearly polarized with parallel polarization. The weakly focused output of the interferometer intersected the supersonic molecular beam sample at a distance of several millimeters from the expansion orifice of the beam.

The molecular beam was formed by entraining the vapor from heated $\left(\mathrm{T}=100^{\circ} \mathrm{C}\right)$ tolane (Sigma) in carrier gas and allowing the mixture to expand continuously through a small orifice $(\sim 50 \mu \mathrm{m}$ in diameter) into vacuum. The carrier gas consisted of helium (for the bare molecule studies) or helium with a small amount of argon or nitrogen mixed in by means of a needle valve arrangement, the total pressure being about 80 psig. Laser-induced fluorescence was collected with an elliptical mirror, filtered spatially and spectrally (with sharp-cut filters) to eliminate scatter, and detected with a photomultiplier. The photomultiplier signal was averaged with a boxcar integrator whose output was stored by a computer as a function of delay between the pump and probe pulses (i.e., the interferometer delay).

Measured RCS-TRFD traces were manipulated numerically so as to eliminate any delay dependence in background fluorescence levels due to fluorescence lifetime effects ${ }^{4}$ (except those on a subnanosecond timescale), delay-line misalignment, laserbeam divergence, etc. To determine rotational constants from the data the traces 
were compared with simulated traces calculated by using the theoretical results of References 6 and 7. These simulations were performed by assuming a temperature of $5 \mathrm{~K}$ and a Gaussian temporal response of $28 \mathrm{ps} \mathrm{fwhm}$.

Fluorescence excitation spectra of the tolane/argon and tolane/nitrogen beams were also measured. The excitation pulses were generated by frequency doubling (with an auto-tracking Spectra-Physics WEX-1c) the output of a Nd:YAG-pumped dye laser (Spectra-Physics PDL-3, Rhodamine 590 as dye). The excitation pulses were attenuated significantly before being directed to the molecular beam. Fluorescence was detected and averaged with the same apparatus as used in the TRFD experiments.

\section{RESULTS}

\section{A. Tolane}

The vibronic spectroscopy of tolane has been investigated by Okuyama, et al. ${ }^{2}$ Several assignments were made by them of bands in the fluorescence excitation spectrum of the molecule. In particular, it was concluded that two electronic states could be reached by single-photon excitation from the ground state in the region 35,051$35,589 \mathrm{~cm}^{-1}$. One electronic state of $B_{2 u}$ symmetry (the axis convention employed herein is given in Figure 1a) was invoked in assigning two of the more intense bands: the $a$ and $b$ bands at 35,051 and $35,281 \mathrm{~cm}^{-1}$, respectively. A second electronic state of $B_{1 u}$ symmetry was invoked in assigning the two most intense $A$ and $B$ bands at 35,248 and $35,589 \mathrm{~cm}^{-1}$, respectively. We have measured RCS traces for these four major bands of bare tolane.

Figure 1 shows the measured RCS-TRFD trace and the simulated fit for excitation of the $b$ band. The trace clearly exhibits seven rotational coherence transients aside from the prominent feature at $t=0$. There are two large negative transients at 2,086 and $4,180 \mathrm{ps}$ and three large positive going features at 1,043, 3,136 and 5,216 ps. Given the equal spacing $(\sim 1,043 \mathrm{ps})$ of these five large features, it is clear that they are of the same type. Furthermore, their polarity characteristics and the fact that the positive-polarity features are smaller than the negative-polarity ones dictate that they be assigned as "J-type" transients. ${ }^{3}$ These transients occur at times $t \simeq m / 2(B+C)$ for near prolate symmetric tops, $m$ being an integer. Two smaller features are also visible in the experimental results of Figure 1, a negative-going transient at 4,292 ps and a positive one at 5,355 ps. These are "C-type" transients, which occur at $t \simeq m / 4 C$. By comparing the observed trace with simulated ones we derive values for $B+C$ and $C$ of $0.478 \pm 0.002$ and $0.229 \pm 0.001 \mathrm{GHz}$, respectively.

Besides rotational constants, the direction of the transition dipole associated with band $b$ can be obtained from the experimental trace of Figure 1. The characteristics of the RCS trace, particularly the magnitudes of the transients relative to the feature at $t=0$ and the absence of transients other than $\mathrm{J}$ - and C-type, clearly indicate that it is associated with an $A$-axis polarized vibronic transition in the near-prolate tolane molecule. ${ }^{3}$ Assuming $D_{2 h}$ point-group symmetry to hold for tolane, this means that 
(a)
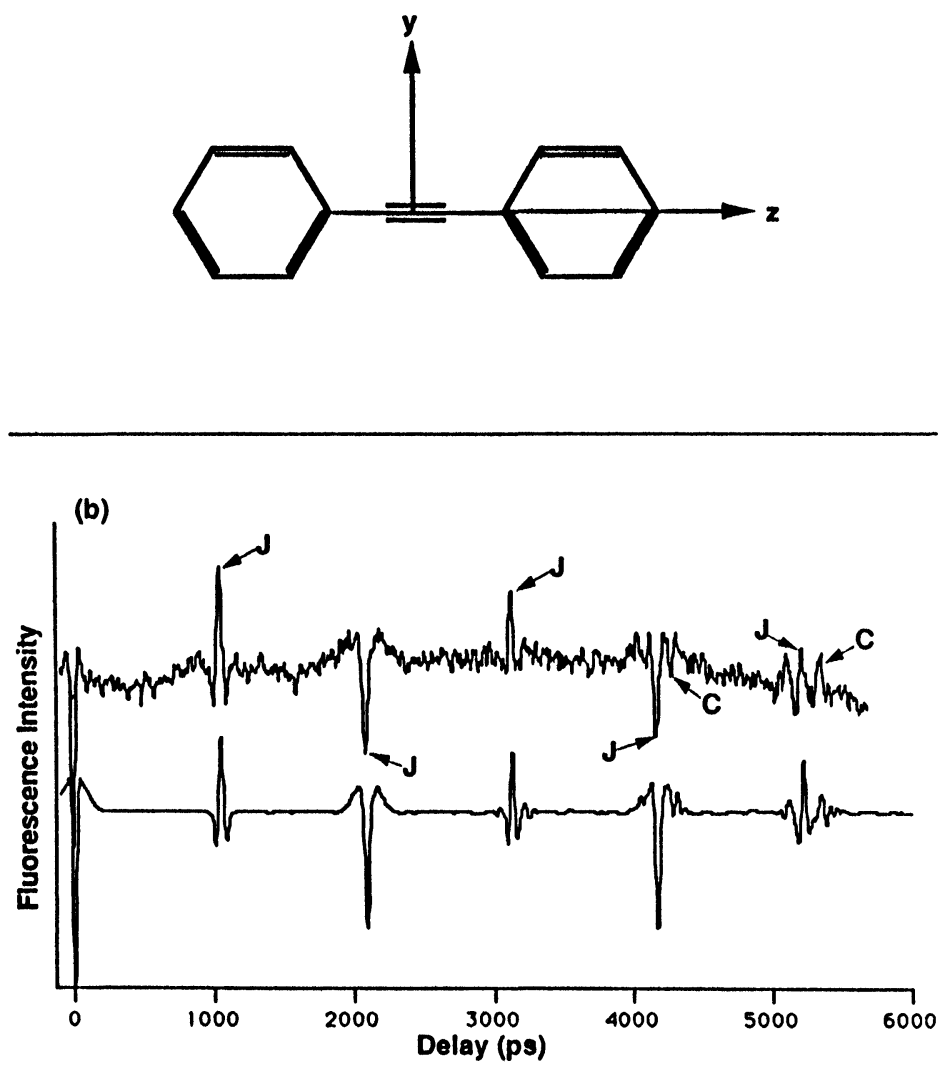

Figure 1 (a) The tolane-fixed axis convention used herein. (b) Top-Measured RCS-TRFD trace for band $b$ of tolane (see Reference 2). Features labeled with J's (C's) have been assigned as J-type (C-type) rotational coherence effects (see text). Bottom-Simulated trace that best fits the measured trace. The rotational constants for the calculated trace were taken as $2.896,0.249$, and $0.229 \mathrm{GHz}$, respectively, for $A, B$, and $C$. The transition dipole was taken to be along the $A$ principal axis of tolane (i.e., the $z$ axis in Figure 1a).

band $b$ comes from a transition between the ground-state and an excited vibronic state having $B_{1 u}$ vibronic symmetry.

Figure 2 shows RCS-TRFD traces for the $b, A$, and $a$ bands of tolane. It is clear that the three traces are very similar and exhibit the features that were discussed above in conjunction with band $b$. Because of this we also assign $B_{1 u}$ vibronic symmetries to the excited states associated with the $A$ and $a$ bands. Moreover, we note that the $B+C$ and $C$ rotational constants derived from all three traces are identical within our measurement uncertainty.

Figure 3a shows the RCS-TRFD trace obtained upon excitation of the $B$ band of tolane. This trace is significantly different from those in Figure 2. The J-type transients are still present and occur in the same positions as before. However, there are other, small transients present in the band- $B$ trace which appear at early times 


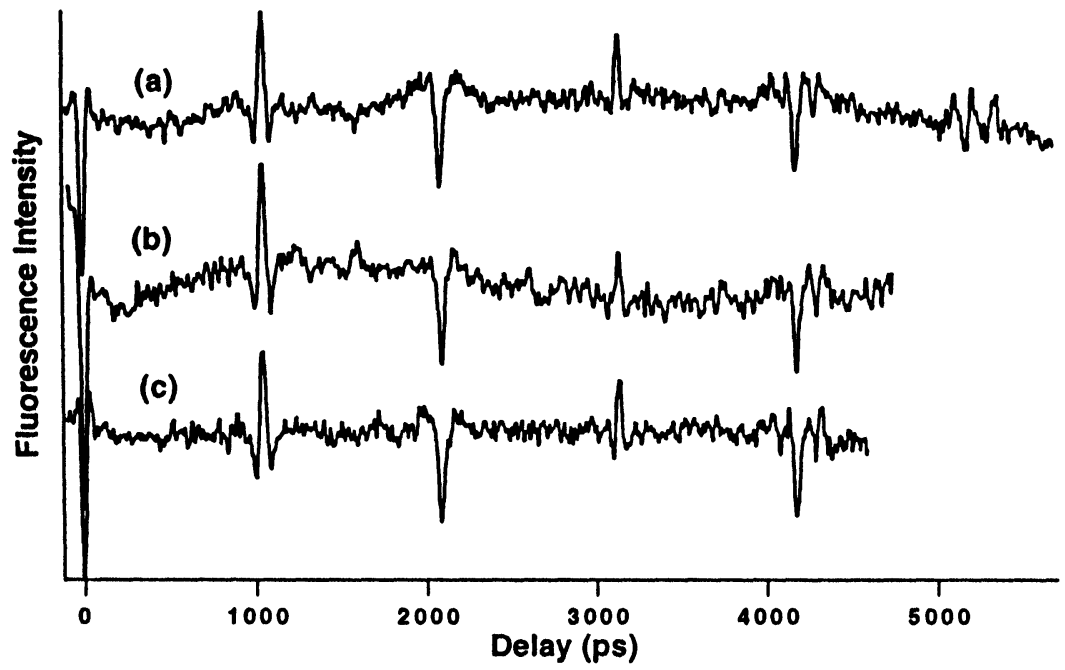

Figure 2 Comparison of measured RCS-TRFD traces for different bands in jet-cooled tolane. (a) Band $b$. (The trace is the same as that in Figure 1b-top.) (b) Band A. (c) Band $a$.

$(t \leq 500 \mathrm{ps})$. In addition, the $t=0$ feature for the trace is considerably larger than the analogous features in the traces of Figure 2. This can be seen by the comparison in Figure $3 \mathrm{~b}$, which shows the trace from the $B$ band and that from the $a$ band on the same absolute-depletion scale. There are two possible explanations for the differences in the $B$ band trace. One is that the transition dipole direction associated with the band is along the $B$ principal axis (in-plane, short-axis) of tolane. This would account for the additional transients in the trace (such could be attributed to "K-type" RCS transients ${ }^{3}$ ). However, this interpretation cannot account for the markedly increased magnitude of the $t=0$ depletion in the $B$-band trace. A second explanation is more likely: namely, that the excited-state prepared by $B$-band excitation decays by a fast (ca. $10 \mathrm{ps}$ ) dynamical process. Such a process will increase the depletion at $t=0$ and might also be expected to produce extra transients-that is, recurrences (quantum beats) associated with the dynamical process. ${ }^{8}$ Notably, a fast decay of the initially prepared state associated with band $B$ does not imply that RCS transients will not be observed for that band. This is because the TRFD method is a probe of rotational coherences in both the vibronic states connected by the pump pulse. ${ }^{7}$ When the excited vibronic state is short-lived enough that excited-state rotational coherences cannot be observed (since they decay away too quickly), ground-state coherences can still enter into TRFD traces (since they decay at the decay rate of the ground vibronic state). ${ }^{7}$ Thus, the RCS transients in Figure $3 \mathrm{a}$ and $3 b$-bottom correspond to ground-state rotational coherences and depend on groundstate rotational constants of tolane. A second point to be made concerning our interpretation of the $B$-band trace is that fast decay of the laser-excited state does not imply a low quantum yield for fluorescence. This is because the decay of the initial state might well populate "relaxed" states that have appreciable fluorescence yields. The point is that the large intensity of band $B$ in the fluorescence excitation 

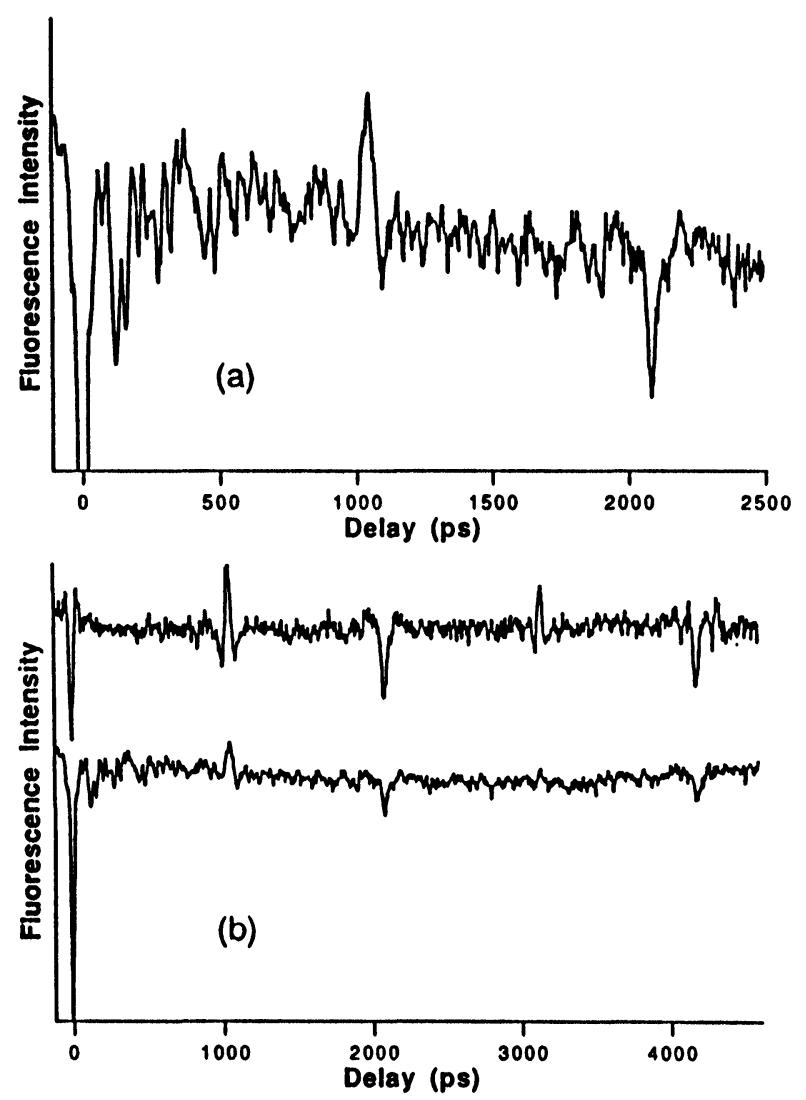

Figure 3 (a) An expanded view of the RCS-TRFD trace measured for band $B$ of tolane. (b) A comparison of RCS-TRFD traces measured for band $B$ (bottom) and band $a$ (top) of tolane. The two traces are plotted such that a given fluorescence depletion magnitude (in \% of the total signal) will have the same amplitude in either trace. Note the relative magnitudes of the $t=0$ features and the fact that the RCS transients at $t>0$ are about one-half as big in the lower trace as in the upper.

spectrum of tolane ${ }^{2}$ is not inconsistent with the fast-decay interpretation invoked here. Further discussion of the fast decay of band $B$ is given in section $\mathrm{V}$.

\section{B. Tolane-Ar}

The fluorescence excitation spectrum of the tolane/argon beam in the region of the $A$ band of tolane is shown in Figure 4-top. Figure 4-bottom is an excitation spectrum in the same region of uncomplexed tolane. One sees that additional peaks appear in the spectrum when argon is present. We point out two features in particular, one at $-46 \mathrm{~cm}^{-1}$ and one at $-90 \mathrm{~cm}^{-1}$ with respect to band $A$ of bare tolane. Following the vibronic red-shift rules for aromatic-(rare gas) ${ }_{n}$ species (e.g., Reference 9) we assign these bands to tolane-Ar and tolane- $\mathrm{Ar}_{2}$.

Figure 5 shows the measured and fit RCS-TRFD traces for excitation of the tolane-Ar band at $35,202 \mathrm{~cm}^{-1}$. It is clear from the measured trace that four J-type 


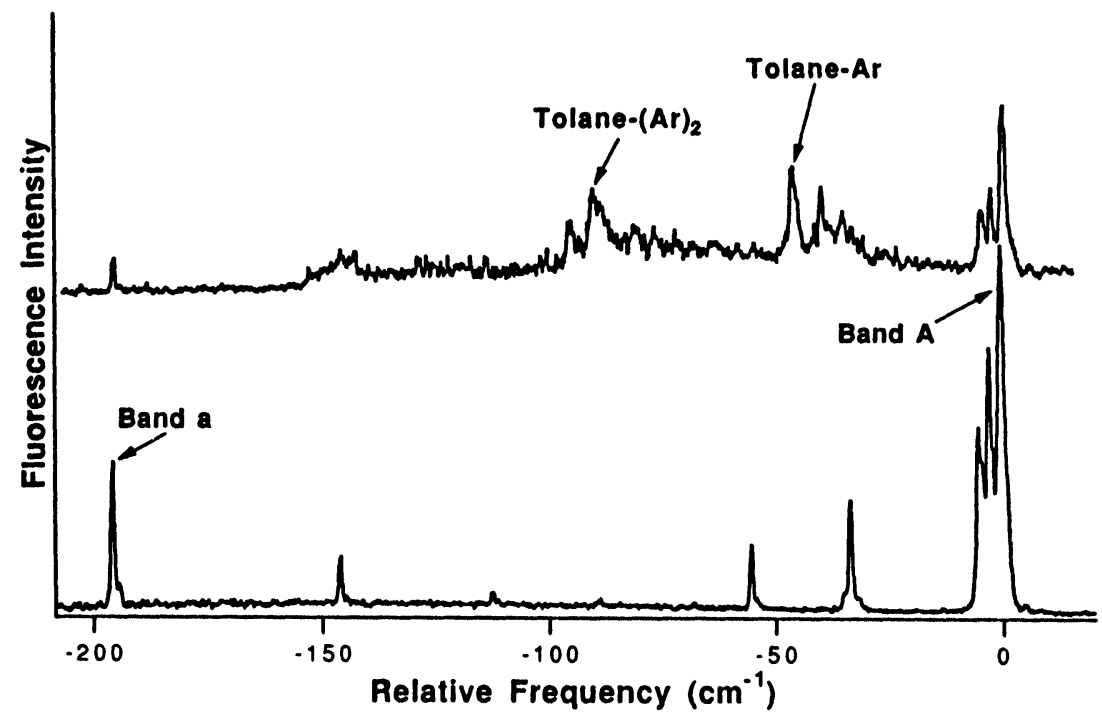

Figure 4 Top-Fluorescence excitation spectrum of a free-jet expansion consisting of tolane and argon in helium. Bottom-Fluorescence excitation spectrum of a free-jet expansion of tolane in helium. The zero of the frequency scale is the position of band $A$ of tolane $\left(35,248 \mathrm{~cm}^{-1}\right.$-see Reference 2$)$.

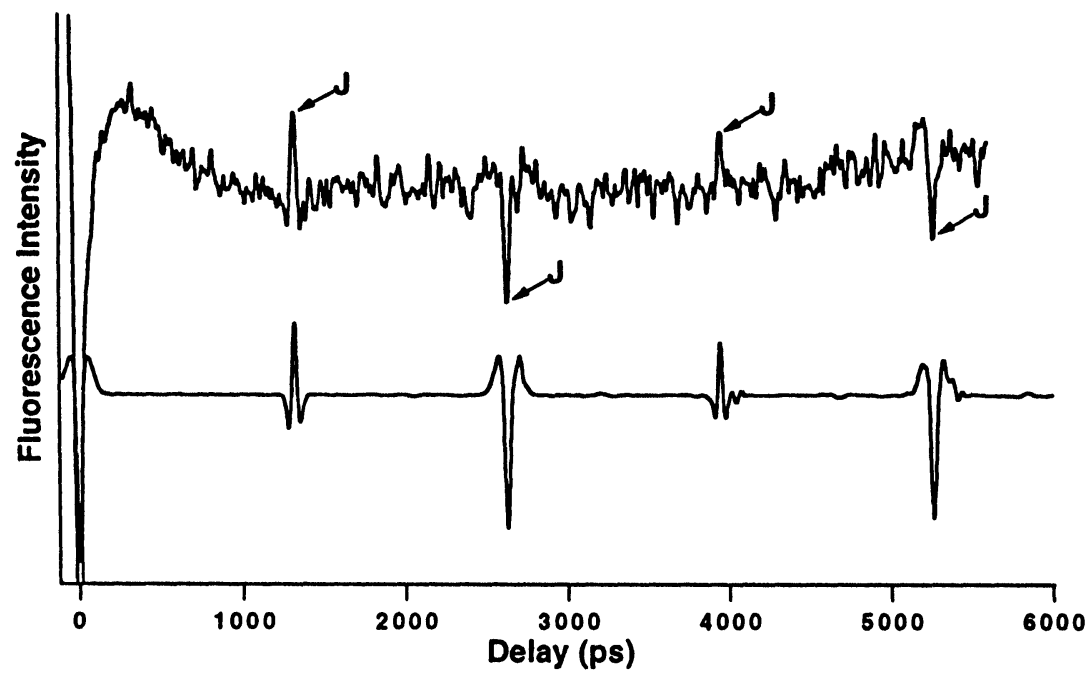

Figure 5 Top-Measured RCS-TRFD trace for the band at $-46 \mathrm{~cm}^{-1}$ in Figure 4-top, which has been assigned to tolane-Ar. Bottom-Calculated trace which best fits the experimental trace above. The rotational constants for the calculated trace were taken as $0.915,0.380$, and $0.010 \mathrm{GHz}$, respectively, for $A$, $B+C$, and $B-C$. The transition dipole was taken to be along the $A$ axis of the species.

transients are present at $1314,2628,3946$ and 5260 ps. Based on these positions, the $B+C$ rotational constant is $0.380 \pm 0.002 \mathrm{GHz}$. Because there are no C-type transients present in this trace, it is not possible to derive from it a value of $C$ 
for tolane-Ar. However, it is possible from the results to put an upper limit on $B-C$. This can be done by comparing the measured trace to simulated traces in which $B+C$ is held constant and $B-C$ is varied. By doing this we find $B-C$ $\leq 0.010 \mathrm{GHz}$.

One may notice in Figure 5 that the feature at $t=0$ is broadened relative to the $t=0$ feature of the calculated trace. In addition, the RCS transients are smaller in magnitude than would be expected for an $A$-type (or near $A$-type) transition in this species. We believe this behavior to be due to some rapid dynamical process in the excited-state manifold of tolane-Ar subsequent to excitation at $35,202 \mathrm{~cm}^{-1}$. Figure 6 shows an expanded view of the $t=0$ transient of the trace in Figure 5-top. Also shown is a measured $t=0$ feature (from a bare tolane trace) that is solely a manifestation of rotational coherence effects. One sees that the tolane-Ar transient extends to significantly longer time. An exponential fit of the feature at later times indicates a lifetime for the excited state of $\sim 100$ ps. (Such a fit is also shown in Figure 6.) Possible processes contributing to this rapid decay are discussed in section $\mathrm{V}$.

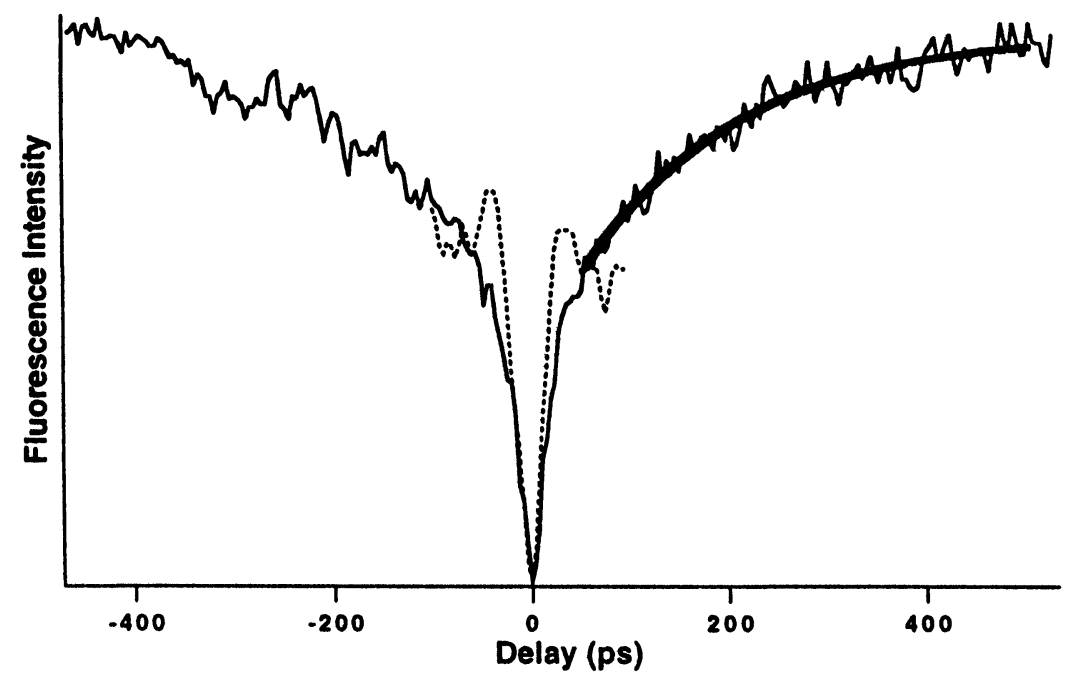

Figure 6 Thin line-The RCS-TRFD trace of Figure 5-top (tolane-Ar) in the $t=0$ region. Dotted line-RCS-TRFD trace of bare tolane in the $t=0$ region. Bold line-Exponential fit of the RCS-TRFD trace for tolane-Ar.

One last point of note about the tolane-Ar trace of Figure 5 is that there are observable RCS transients at times longer than $5,000 \mathrm{ps,} \mathrm{even} \mathrm{though} \mathrm{the} \mathrm{excited}$ state prepared by the pump laser pulse lives for only $100 \mathrm{ps}$. The existence of such transients can be explained by the same argument used in conjunction with band $B$ of bare tolane. That is, TRFD probes both ground- and excited-state rotational coherences. ${ }^{7}$ The former will persist even in situations where the latter decay rapidly due to a short excited-state lifetime. Given this, we assign the RCS transients in 
Figure 5 to ground-state rotational coherences and take the rotational constants derived from the trace to be ground-state values.

\section{Tolane- $\mathrm{N}_{2}$}

The fluorescence excitation spectrum of the tolane/ $\mathrm{N}_{2}$ beam is shown in Figure 7 . As was the case with argon, two features appear in the spectrum which can be attributed to tolane- $\mathrm{N}_{2}$ and tolane- $\left(\mathrm{N}_{2}\right)_{2}$. The band assigned to tolane- $\mathrm{N}_{2}$ is shifted $-43 \mathrm{~cm}^{-1}$ from the tolane $A$ band and that for tolane- $\left(\mathrm{N}_{2}\right)_{2}$ is shifted $-77 \mathrm{~cm}^{-1}$. The feature we assign to tolane- $\left(\mathrm{N}_{2}\right)_{2}$ is very broad. This may reflect the presence of several isomers of this species or that of other tolane- $\left(\mathrm{N}_{2}\right)_{n}$ clusters having vibronic resonances in this spectral region.

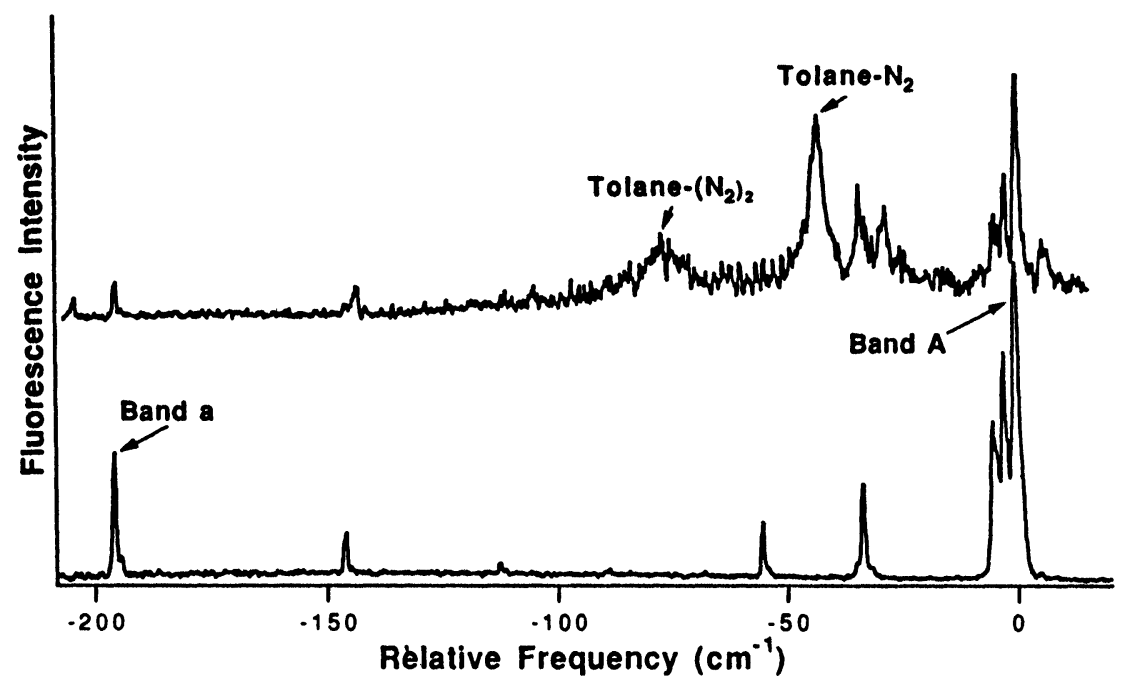

Figure 7 Top-Fluorescence excitation spectrum of a free-jet expansion consisting of tolane and $\mathrm{N}_{2}$ in helium. Bottom-Fluorescence excitation spectrum of a free-jet expansion of tolane in helium. The zero of the frequency scale is the position of band $A$ of tolane.

Figure 8 shows a RCS-TRFD trace corresponding to the $35,205 \mathrm{~cm}^{-1}$ band assigned to tolane- $\mathrm{N}_{2}$. Present in the trace are essentially the same features that appear in the tolane-Ar trace of Figure 5. Now, though, the J-type transients occur at 1,246, 2,480 and 3,743 ps. Fitting the measured trace yields $B+C=0.401 \pm 0.002 \mathrm{GHz}$ and $B-C \leq 0.015 \mathrm{GHz}$ for tolane- $\mathrm{N}_{2}$. One notes again that the $t=0$ feature in the trace is broader than would be expected if it were due entirely to rotational coherence effects. Indeed, an exponential fit to the long-time tail of the $t=0$ transient (analogous to that of Figure 6) yields a lifetime for the initially excited state of roughly $50 \mathrm{ps}$. Thus, as with tolane-Ar, we find evidence for the presence of fast excited-state relaxation in tolane- $\mathrm{N}_{2}$. This also implies that the RCS transients in Figure 8 reflect ground-state rotational coherences entirely. 


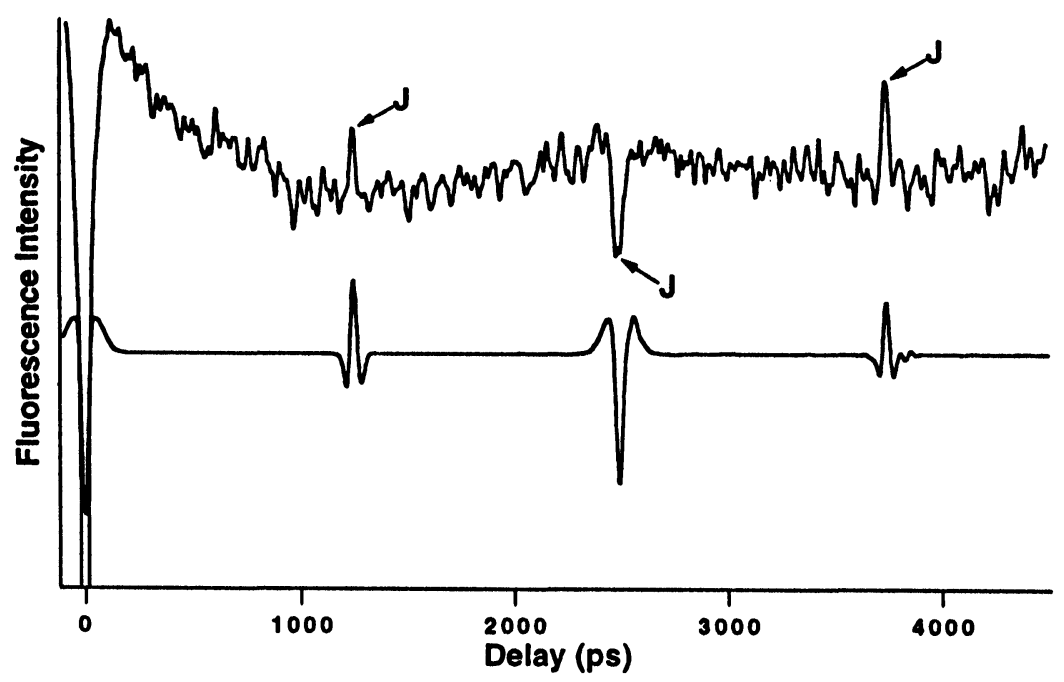

Figure 8 Top-Measured RCS-TRFD trace for the band at $-43 \mathrm{~cm}^{-1}$ in Figure 7-top, which has been assigned to tolane- $\mathbf{N}_{2}$. Bottom-Calculated trace which best fits the experimental trace above. The rotational constants for the calculated trace were taken as $1.135,0.401$, and $0.010 \mathrm{GHz}$, respectively, for $A$, $B+C$, and $B-C$. The transition dipole was taken to be along the $A$ axis of the species.

\section{STRUCTURE ANALYSIS}

\section{A. Tolane}

The work of Okuyama, et al. ${ }^{2}$ on the vibronic spectroscopy of tolane provides good evidence that the species is planar in the gas phase. This conclusion is reinforced by the RCS results that we have presented above. In particular, the following relation can be shown to be true for tolane:

$$
\frac{B-C}{B+C}=\frac{\left(I_{2}-I_{1}\right) \cos \theta}{\left(M r^{2}\right) / 2+\left(I_{1}+I_{2}\right)}
$$

In this relation $B$ and $C$ are the rotational constants of tolane and $\theta$ is the angle between the planes of the phenyl rings in the molecule. The other parameters refer to constants associated with the two identical moieties formed by bisecting tolane's triple bond: (1) $M$ is the mass of each moiety ( $89 \mathrm{amu}),(2) r$ is the distance between their two centers-of-mass $\left(r=6.19 \AA\right.$ assuming standard bond lengths and angles $\left.{ }^{10}\right)$, (3) $I_{1}$ is the moment of inertia of either moiety about the principal axis of that moiety that lies in the plane of its phenyl ring and is perpendicular to the $C-C$ single-bond axis $\left(I_{1}=168 \mathrm{amu}-\AA^{2}\right.$ from standard bond lengths, etc. $\left.{ }^{10}\right)$, and (4) $I_{2}$ is the analogous moment of inertia of either moiety about that moiety's principal axis that is perpendicular to its ring plane $\left(I_{2}=256 \mathrm{amu}-\AA^{2}\right)$. With the measured rotational constants and the values given above we find that $\cos \theta=1.02 \pm 0.05$, which is consistent with a planar structure for tolane. 


\section{B. Tolane-Ar}

To obtain structural information on tolane-Ar from the RCS results, we make the usual assumption that the geometries of the monomers do not change upon complex formation. Unfortunately, even with this assumption, the RCS results for tolane-Ar are not sufficient to specify its structure completely. A second assumption is required. Thus, we assume that the Ar atom is located above the plane of tolane at a distance of $3.5 \AA$. This assumption is one that is consistent with the significant body of knowledge on the interaction geometry of an Ar atom with single-ring aromatics. ${ }^{11}$ With this structural degree of freedom fixed, we fit the observed rotational constants of tolane-Ar in a nonlinear least-squares procedure that employed the two other geometrical degrees of freedom as variable parameters. The resulting, best-fit structure is shown in Figure 9. As shown in the figure, the argon atom is above the plane of a phenyl ring, but is shifted slightly away $(0.3 \AA)$ from the center of that ring toward the acetylenic group of the molecule.

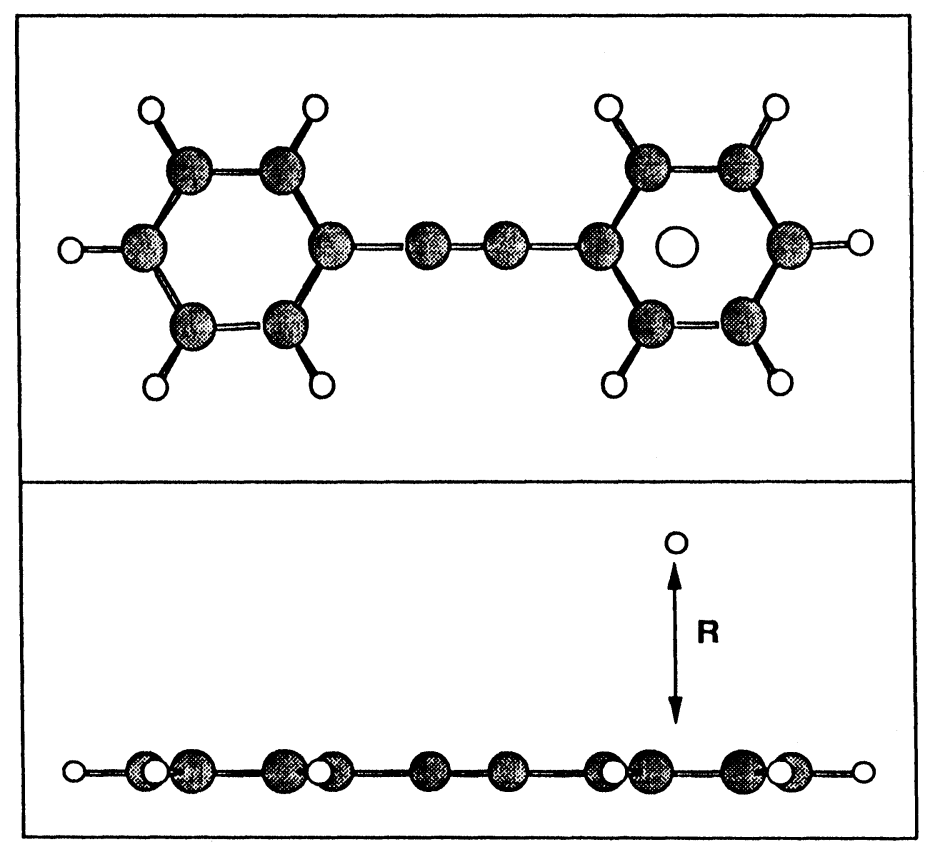

Figure 9 Two views of a tolane-Ar geometry that is consistent with the RCS-measured rotational constants for the species. $\mathrm{R}$ was fixed at $3.5 \AA$.

\section{Tolane- $-N_{2}$}

Analysis of the structure of tolane- $\mathrm{N}_{2}$ was performed in a manner analogous to the analysis of the tolane-Ar structure. Once again monomer moieties were assumed to be unchanged upon complexation and the $\mathrm{N}_{2}$ moiety was taken to be above the plane of tolane at a distance of $3.5 \AA$, consistent with the geometry of benzene- $\mathrm{N}_{2}{ }^{12} \mathrm{~A}$ 
further assumption was also required, namely, that the $\mathrm{N}_{2}$ axis lies parallel to the tolane plane in the complex. This assumption is also consistent with structural results on benzene- $\mathrm{N}_{2}$. With these assumptions, the two remaining structural degrees of freedom are the $y$ and $z$ coordinates of the $\mathrm{N}_{2}$ center-of-mass with respect to the tolane-fixed axis system of Figure 1a. (The angle between the bond axis of $\mathrm{N}_{2}$ and the $z$ axis is also a variable, yet has little effect on the rotational constants. Moreover, it is likely that the vibrationally averaged structure samples all such angles with similar probability. ${ }^{12}$ ) Nonlinear least-squares fits to the observed rotational constants obtained by using these center-of-mass coordinates as adjustable parameters yielded structures such as the one shown in Figure 10 as best-fit ones. Again, one finds that the small species binds above a phenyl ring at a position slightly displaced away from the center of the ring toward the acetylenic group.

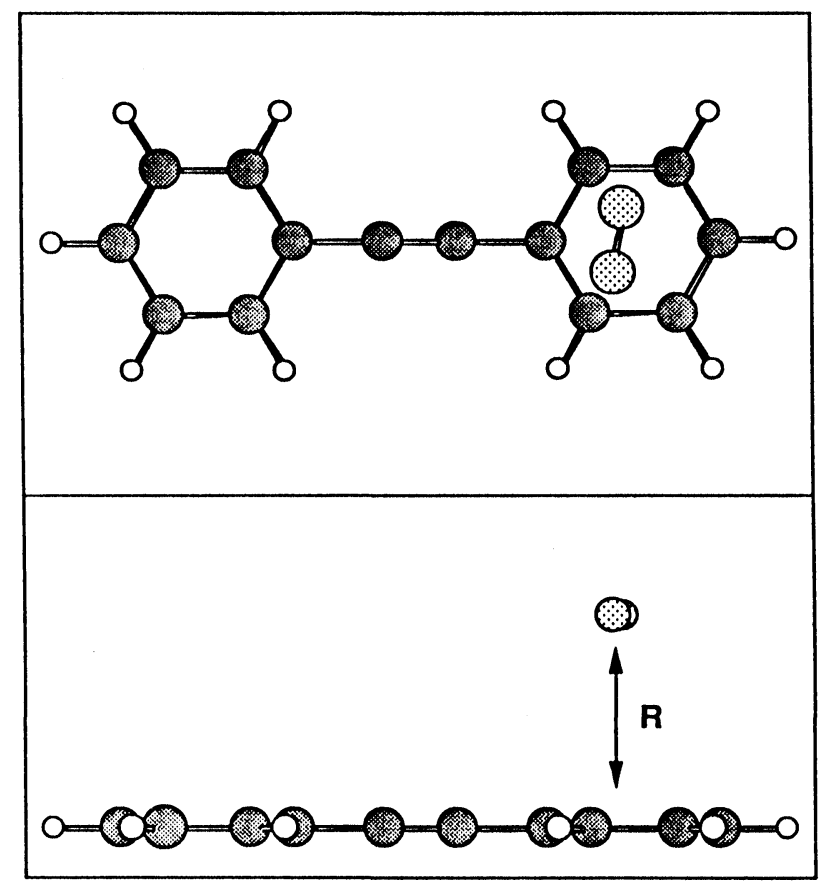

Figure 10 Two views of a tolane- $\mathrm{N}_{2}$ geometry that is consistent with the RCS-measured rotational constants for the species. $\mathrm{R}$ was fixed at $3.5 \AA$.

\section{DISCUSSION}

\section{A. Excited States of Tolane}

The results of Reference 2 provide good evidence for the presence of vibronic bands from three excited electronic states in the excitation-energy range from 
34,900 to $35,600 \mathrm{~cm}^{-1}$ in tolane. Fluorescence excitation spectra indicate that bands $A$ and $B$ belong to the same electronic state since the same low-frequency progression is built off them both. The low frequency progressions built off of bands $a$ and $b$ are also identical, yet they differ from those associated with bands $A$ and $B$. This is a good indication that the two pairs of bands arise from different electronic states. Finally, the two-photon resonant, four-photon ionization spectrum of Reference 2 reveals bands that do not appear at all in the fluorescence excitation spectrum. With their experimental information and the results of calculations ${ }^{13}$ pertaining to excited-state energies, the authors of Reference 2 concluded that bands $A$ and $B$ belong to an electronic state of $B_{1 u}$ symmetry, bands $a$ and $b$ belong to a state with $B_{2 u}$ symmetry, and the two-photon-resonant bands belong to a state with $A_{g}$ symmetry. The RCS results on bare tolane lead to one unequivocal conclusion regarding the symmetries of vibronic states, that is, that the $A, a$, and $b$ bands are associated with excited states of $B_{1 u}$ vibronic symmetry. The results also indicate that the excited state associated with band $B$ also has $B_{1 u}$ vibronic symmetry but is subject to a rapid decay process. These conclusions are consistent with the assignment ${ }^{2}$ of the $a$ and $b$ bands as vibronically induced ones that involve excitation into the manifold of a $B_{2 u}$ excited electronic state. In this interpretation, states with $b_{3 g}$ vibrational symmetry in the manifold of a low oscillator-strength $B_{2 u}$ electronic state gain intensity by vibronic coupling to a nearby $B_{1 u}$ electronic state. The RCS-based conclusions are also consistent with the assignment ${ }^{2}$ of the $A$ band as the $0_{0}^{0}$ band of a $B_{1 u} \leftrightarrow S_{0}$ electronic transition. Presumably, this is the same $B_{1 u}$ electronic state from which the $a$ and $b$ bands borrow oscillator strength. Finally, the indication that band $B$ produces an excited state of $B_{1 u}$ vibronic symmetry is consistent with the assignment of this band as one involving a totally symmetric vibrational band built off band $A$ as origin.

It is of interest to consider the possible source of rapid decay of the excited-state reached through band $B$ in tolane. This decay and subsequent, low-amplitude quantum beats (see Figure 3) could be due to intramolecular vibration energy redistribution (IVR) within the $B_{1 u}$ electronic-state manifold (to which bands $B$ and $A$ have been assigned). This is probably not the source of the behavior, however, because band $B$ is only $340 \mathrm{~cm}^{-1}$ higher in energy than the $A$ band, which we have presumed to be the $0_{0}^{0}$ band of the $B_{1 u} \leftarrow S_{0}$ transition. This amount of vibrational energy is most likely not large enough to give rise to dissipative IVR $^{8}$ in the species. (For example, greater than $\sim 1,000 \mathrm{~cm}^{-1}$ vibrational energy is required for dissipative IVR in the $S_{1}$ manifold of the similar $t$-stilbene molecule. ${ }^{8,14}$ ) On the other hand, as noted above, the evidence indicates that two singlet electronic states lie below the $B_{1 u}$ state in tolane. Vibrational levels of these states provide a manifold of states with which levels of the $B_{1 u}$ state can couple. In other words, internal conversion out of the $B_{1 u}$ manifold to these other electronic state manifolds might be expected. It is to such a process that we attribute the rapid decay observed after $B$-band excitation. Further, the observation of quantum beats accompanying this decay indicates that the process corresponds to the "intermediate case" of radiationless transition theory. ${ }^{15}$ That is, the number of states coupled to the laser-prepared state is small 
enough that recurrences (albeit small ones) in the population of that state occur on the timescale of the experiment.

\section{B. Structure and Dynamics of Tolane-Ar and $-N_{2}$}

The notable feature about the RCS-derived geometries of tolane-Ar and tolane- $\mathrm{N}_{2}$ is the fact that the binding of the smaller species to tolane occurs above a phenyl ring, yet displaced along the $z$ axis away from the center of that ring toward the acetylenic moiety. This same geometrical feature also characterizes the similar complex composed of Ar and $t$-stilbene. ${ }^{16}$ As pointed out in Reference 16, this kind of geometry is rather straightforward to rationalize. On the one hand, it is close to the structures that have been determined for single-ring aromatics in binding to small nonpolar species, such structures generally being ones in which the smaller species is bound directly above the center of the phenyl ring. ${ }^{11}$ On the other hand, there is significantly more to the tolane molecule than one phenyl moiety. One expects this other part of the molecule also to have some influence on the binding geometry. Moreover, one expects such influence to be attractive due to long-range dispersion interactions. The effect is to shift the minimum energy position away from the center of a phenyl ring and toward the remainder of the molecule.

It is of interest to compare the experimental structure of tolane-Ar with the minimum-energy geometry calculated by assuming the Lennard-Jones atom-atom potential energy surface given in Reference 17 (variants of which are used extensively in modeling the properties of aromatic-(rare gas) ${ }_{n}$ species). We find (by use of a simplex minimization scheme ${ }^{18}$ ) two energy minima for tolane-Ar. One minimum, with a binding energy of $369 \mathrm{~cm}^{-1}$, puts the Ar atom about $3.06 \AA$ above the triple bond but displaced in the $y$ direction (See Figure 1a) by about $1.8 \AA$ and in $z$ direction by about $0.4 \AA$. This geometry is somewhat analogous to the minimum-energy $t$-stilbene-Ar structure found in Reference 16 by a similar calculation. The second, more stable geometry (binding energy of $392 \mathrm{~cm}^{-1}$ ) has the Ar atom located $3.44 \AA$ directly above the center of a phenyl ring. This latter geometry is fairly close to the one derived from our RCS measurements. However, it does not reproduce the slight displacement of the Ar atom away from the ring center toward the acetylene group.

Finally, we consider the fast excited-state decay processes that we have observed in the $\mathrm{Ar}$ and $\mathrm{N}_{2}$ complexes. At first sight these fast decays might not be expected since they occur subsequent to excitation of what are most likely the $0_{0}^{0}$ bands of a ${ }^{1} B_{1 u} \leftarrow S_{0}$ electronic transition in the complexes. (This assignment follows from the assignment of band $A$ of tolane as such a transition.) With no vibrational energy in the excited state one wonders how the usual fast processes in weakly bound complexes-vibrational energy redistribution and predissociation-can occur. Again, however, one must note the likely presence of at least two tolane-localized, singlet electronic states that are lower in energy than the electronic state to which the complexes are excited. As with band $B$ of bare tolane, these lower-energy, close-lying electronic states can provide a channel for efficient inter-electronic radiationless transitions. It is to just such transitions that we attribute the fast decays in the van der 
Waals complexes. Evidently, the increased density of vibrational states in the complexes relative to that in bare tolane "turns on" these processes at a lower energy in the complexes than that for which they occur in the bare molecule. Unfortunately, with just TRFD data we are not able to say much more about the specific nature of the decay process. It could be solely internal conversion or it could be internal conversion followed by vibrational energy redistribution and/or predissociation of the complex. A more complete study involving the measurement of the decays of dispersed fluorescence bands (e.g., see Reference 19) would help to address this issue.

\section{Acknowledgments}

This work was supported by the US National Science Foundation through Grant No. CHE 91-15656.

\section{References}

1. For example, see the reviews (a) M. Ito. J. Mol. Struct., 177, 191 (1988); (b) M. Ito. J. Phys. Chem., 91, 517 (1987); (c) M. Ito, T. Ebata, and N. Mikami. Ann. Rev. Phys. Chem., 39, 123 (1988).

2. K. Okuyama, T. Hasegawa, M. Ito, and N. Mikami. J. Phys. Chem., 88, 1711 (1984).

3. See P. M. Felker. J. Phys. Chem., 96, 7844 (1992).

4. M. J. Côté, J. F. Kauffman, P. G. Smith and J. D. McDonald. J. Chem. Phys., 90, 2864 (1989); J. F. Kauffman, M. J. Côté, P. G. Smith and J. D. McDonald. J. Chem. Phys., 90, 2874 (1989).

5. L. L. Connell, S. M. Ohline, P. W. Joireman, T. C. Corcoran and P. M. Felker. J. Chem. Phys., 96, 2585 (1992).

6. P. M. Felker and A. H. Zewail. J. Chem. Phys., 86, 2460 (1987).

7. G. V. Hartland, L. L. Connell and P. M. Felker. J. Chem. Phys., 94, 7649 (1991).

8. For example, P. M. Felker and A. H. Zewail. Adv. Chem. Phys., LXX, 265 (1988).

9. J. Bösiger and S. Leutwyler. Chem. Phys. Lett., 126, 238 (1986).

10. For example, see Handbook of Chemistry and Physics, 57th Edition (CRC Press, Cleveland, 1976), p. F-215.

11. For example. D. V. Brumbaugh, J.E. Kenny, and D. H. Levy. J. Chem. Phys., 80, 2256 (1984); Th. Weber, E. Riedle, H. J. Neusser, and E. W. Schlag. Chem. Phys. Lett., 183, 77 (1991); K. Yamanouchi, S. Isogai, S. Tsuchiya, and K. Kuchitsu. Chem. Phys., 116, 123 (1987).

12. Th. Weber, A. M. Smith, E. Riedle, H. J. Neusser and E. W. Schlag. Chem. Phys. Lett., 175, 79 (1990).

13. Y. Tanizaki, H. Inoue, T. Hoshi, and J. Shiraishi. Z. Phys. Chem. Neue Folge, 74, 45 (1971).

14. P. M. Felker, W. R. Lambert, and A. H. Zewail. J. Chem. Phys., 82, 3003 (1985).

15. A. Frad, F. Lahmani, A. Tramer, and C. Tric. J. Chem. Phys., 60, 4419 (1974).

16. J. S. Baskin and A. H. Zewail. J. Phys. Chem., 93, 5701 (1989).

17. S. Leutwyler. J. Chem. Phys., 94, 5099 (1991).

18. W. R. Press, B. P. Flannery, S. A. Teukolsky and W. T. Vettering. Numerical Recipes (Cambridge Univ. Press, Cambridge, 1986).

19. D. J. Semmes, J. S. Baskin, and A. H. Zewail. J. Chem. Phys., 92, 3359 (1990). 